\title{
The Convergence of Deep Learning and Computer Vision: Smart City Applications and Research Challenges
}

\author{
Deep Kothadiya ${ }^{1}$, Aayushi Chaudhari ${ }^{1}$, Ruchita Macwan ${ }^{1}$, Krishna $_{\text {Patel }}^{2}$, Chintan \\ Bhatt $^{1 *}$
}

${ }^{I} U \& P$ U. Patel Department of Computer Engineering, Chandubhai S. Patel Institute of Science and Technology, Charotar University of Science and Technology (CHARUSAT), CHARUSAT campus, Changa 388421, India.

${ }^{2}$ Department of Computer Science \& Engineering, Devang Patel Institute of Advance Technology and Research, Charotar University of Science and Technology (CHARUSAT), CHARUSAT campus, Changa 388421, India.

"Corresponding author. Email: chintanbhatt.ce@charusat.ac.in

\begin{abstract}
In recent years, deep learning strategies started to outshine traditional machine learning methods in a few fields, with Computer Vision being one of the most noticeable ones. The Computer Vision is becoming more suitable nowadays at identifying patterns from images than the human visual cognitive system. It ranges from raw information recording to methods and ideas that span digital image processing, machine learning, and computer graphics. The wide utilization of Computer Vision has attracted many researchers to incorporate their ideas with different fields and disciplines. The era of smart cities has emerged to meet the recent demands of citizens using information and communication technology. This paper reviews research efforts that utilize Deep Learning Frameworks and Computer Vision Applications in support of smart city applications like smart healthcare, smart transportation, smart agriculture, etc. Furthermore, the paper identified key research challenges that emanate from the use of deep learning and computer vision in support of smart city services.
\end{abstract}

Keywords: Agriculture, Computer Vision, Deep Learning, Healthcare, Smart City, Transportation, Video Surveillance.

\section{INTRODUCTION}

Computer Vision is one of the active research fields, especially in the era of AI and robotics science [1]. Computer vision has a very important role to make remarkable improvements [2]. The availability of AI especially deep learning makes revolution from Image Process to Computer vision, with very rich support from Deep learning model like CNN, RNN, LSTM, and many more make CV more applicable in different field of automation like manufacturing, driverless car, healthcare, education, agriculture, satellite images, visual QNA, etc. Name computer vision is very vast in terms of applicability [3]. Computer vision is a combination of object detection, segmentation, reorganization, localization, restoration, etc. [4-6]. With covering such features in computer vision, its applicability became more impactful in the field of face recognition as part of biometrics identification, automated car, AR/VR, disease identification plays a major role in medical science, smart city [7]. Deep learning models can operate complex data from different sources like video, audio, medical images, social media, sensor data (IoT), satellite images [8-11]. Computer vision algorithms are a key element to make smart cities a reality. Smart transportation, energy-saving automatic visual sensor, looking at the infrastructure to alert anomalies activities, counting number of users in infrastructure, taking statistics in peak hour, smart monitoring of human resources as well as infrastructures [12-14]. Computer vision plays a significant role in the development and management of smart cities as they are the "Eyes of the city." In this paper we are going to see below important aspects of smart city in detail like Smart 
Transportation, Smart Healthcare, Smart Agriculture, and Smart Security [15].

\section{COMPUTER VISION FOR SMART CITY}

\subsection{Smart Transportation}

The transportation system is the lifeline of any development and routine of the city [16]. The smooth functioning of the transport system is necessary for any smart city concept. Smart transport offers novel and innovative approaches in different modes of transportation like advanced infrastructure, mobility, traffic control, safety. It provides an advanced, safer, faster, and smart way of travelling. Features of smart transportation systems are public transport management, smart infrastructure management, advanced route management, advance vehicle control and safety, smart payment system, and route information [17-19].
Different technologies in smart transportation like GPS based tracking [20], advance sensing technology, advance video surveillance. Smart transportation system set makeable improvement in smooth transit in city, minimization of pollution, effective parking system, enhance security, utilization of resources [21-25]. Smart Transportation mainly divide in Safety, Efficiency, Security also known as safe secure and effective transportation. Safety in transportation involves applications like Lane Detection, Pedestrian Detection and Driver Monitoring. Efficiency cover application like Traffic flow control, adaptive driving \& warning system, while Security is covered by advance traffic surveillance [26-29]. Figure 1 shows different computer vision technique which are used in different domain of smart transportation. Smart transportation is not limited to listed domain, there is also minor domains like government rules for transportation [30], vehicle support facility, transportation payment facilities

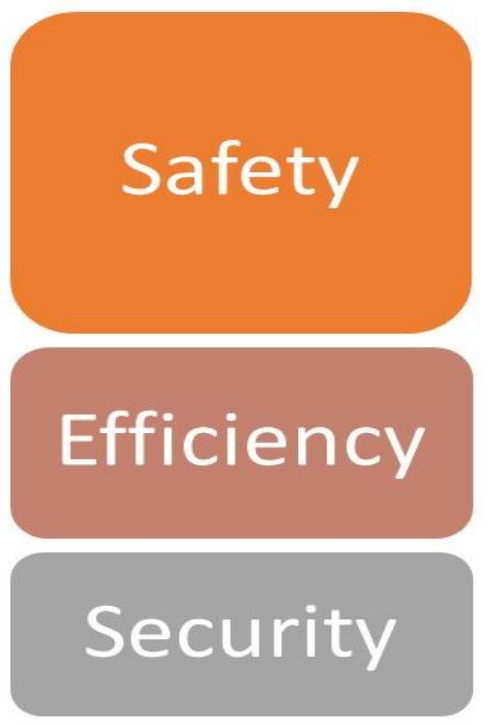

- Edge Detection

- Point Estimation

- Point Segmentation

- Semantic Segmentation

- Drowsiness Detection

- Head Pose Estimation

- Object Recognition

- Object Detection

- Semantic Segmentation

- Object Recognition

- Object Detection

- Text Recognition

- Object Recognition

- Object Detection

- Text Recognition

Figure 1 Pictorial representation of Computer Vision Techniques for Smart Transportation

\subsection{Smart Healthcare}

Smart healthcare includes various health parameters [31]; one of them includes a health monitoring system, which detects the motion of the human body using various techniques like vision-based and sensor-based detection for identifying abnormal activities of a patient to stop unexpected death of humans because of various illness factors. Motion detection is one of the most important technologies in building intelligent healthcare [32]. There are various characteristics for patient monitoring solutions in healthcare which will be useful in upcoming 5-10 years [33]. This characteristic is based on various categories like medium, sensor-based, application based and type of camera required for monitoring [34]. Figure 2 depicts the characteristics in various categories. Motion detection falls mainly into two major categories, visionbased, and sensor-based detection. We can have the vision-based and sensor-based motion detection methods to recognize the fall detection and identify the movements of the patient [35]. 
Medium: Wired/Wireless

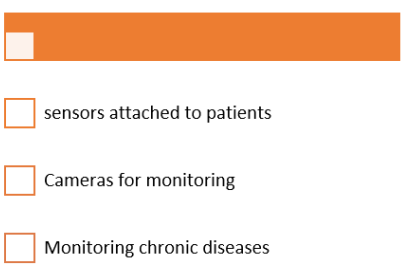

\section{Application: Android/IOS}

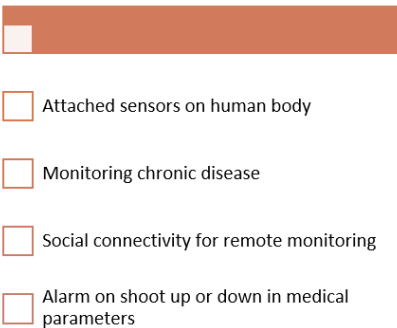

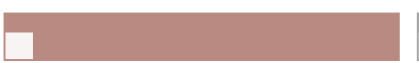

$\square$ Sensors attached on patient's body

Measuring medical parameters

Alarm for sending alert to doctor
Cameras: Normal/HD

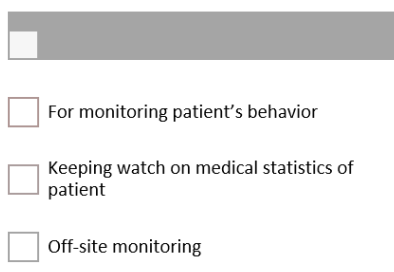

Figure 2 Pictorial representation of Key Characteristics for Patient Monitoring in Span of Upcoming 5 Years

\subsection{Smart Video Surveillance}

Importance of the video surveillance recognition of suspected human activities is to avoid robbery cases [36], objects which are abandoned by terrorists for explosive attacks, mischiefs [37], fights between people and personal attack on someone in the many different places such as banks [38], hospitals, shopping area, parking space, public transport stations, airports, colleges, cities, etc. [39].

Table 1. Approaches for Suspicious Activity Recognition and Detection

\begin{tabular}{|c|c|c|c|}
\hline Works/ Author & Datasets & Classification methods & Result discussion \\
\hline Tian et al. (2012) [39] & $\begin{array}{l}\text { Video Sequences of } \\
\text { PETS2006, i-LIDS }\end{array}$ & $\begin{array}{l}\text { Region Growing and Edge } \\
\text { Energy }\end{array}$ & $\begin{array}{l}\text { Shows poor performance in } \\
\text { low contrast video sequences, } \\
\text { for instance white bottles in } \\
\text { white background. }\end{array}$ \\
\hline Zin et al. (2012a) [40] & $\begin{array}{l}\text { PETS } 2006 \text { and Own } \\
\text { dataset }\end{array}$ & Rule Based Classifier & $\begin{array}{l}\text { Able to detect tiny objects } \\
\text { from video sequences }\end{array}$ \\
\hline Fan and Pankanti (2012) [41] & i-LIDS, AB-L1 and ABL2 & $\begin{array}{l}\text { Structure Similarity and } \\
\text { Region Growing }\end{array}$ & $\begin{array}{l}A B-L 1 \text { and } A B-12 \text { shows that } \\
\text { false positive measure is } \\
\text { reduced by } 6 \% \text { and } 3 \% \\
\text { respectively }\end{array}$ \\
\hline SanMiguel et al. (2012) [42] & ASODds dataset (2011) & $\begin{array}{l}\text { Boundary spatial color } \\
\text { contrast }\end{array}$ & $\begin{array}{l}\text { Proven that feasible for real - } \\
\text { time video sequences. }\end{array}$ \\
\hline Tripathi et al. (2013) [43] & $\begin{array}{l}\text { PETS } 2006 \text { and PETS } \\
2007\end{array}$ & $\begin{array}{l}\text { Edge based object } \\
\text { recognition }\end{array}$ & $\begin{array}{l}\text { Achieved very good accuracy } \\
\text { for both the dataset } \\
\text { respectively } 84.71 \% \text { and } 100 \\
\% \text {. }\end{array}$ \\
\hline Sajith and Nair (2013) [44] & $\begin{array}{l}\text { PETS } 2006 \text { and PETS } \\
2007\end{array}$ & $\begin{array}{l}\text { Neural Network classifier } \\
(\mathrm{NN}) \text { and HOG descriptor }\end{array}$ & $\begin{array}{l}\text { From PETS 2006, detected a } \\
\text { static person as an } \\
\text { abandoned object }\end{array}$ \\
\hline Ferryman et al. (2013) [45] & PETS 2006 & $\begin{array}{l}\text { Logic based inference } \\
\text { engine }\end{array}$ & - \\
\hline
\end{tabular}




\begin{tabular}{|c|c|c|c|}
\hline Chitra et al. (2013) [46] & PETS 2006 & $\begin{array}{l}\text { Support Vector Machine } \\
\text { (SVM) }\end{array}$ & - \\
\hline Nam (2016) [47] & $\begin{array}{l}\text { PETS 2006, PETS } 2007 \\
\text { and i-LIDS }\end{array}$ & Spatio-Temporal & $\begin{array}{l}\text { Achieved for i-LIDS-Precision- } \\
\text { 98.88\%, Recall-82.28\%, F- } \\
\text { measure-82.64\% }\end{array}$ \\
\hline Virender Singh (2020) [48] & $\begin{array}{l}\text { UCF-Crime Dataset, } \\
\text { Annotation, } \\
\text { Preprocessing Testing } \\
\text { Set }\end{array}$ & CNN and RNN & Achieved accuracy about 96\% \\
\hline Amrutha (2020) [49] & KTH & CNN, LSTM, ReLU & $\begin{array}{l}\text { Gained accuracy about } \\
87.15 \%\end{array}$ \\
\hline Rohit (2020) [50] & $\begin{array}{l}\text { Cohn-Kanade, faces94, } \\
\text { faces } 95\end{array}$ & SVM, CNN, RNN & Achieved accuracy about 82\% \\
\hline Arvind (2020) [51] & Own Dataset & $\begin{array}{l}\text { Local binary pattern } \\
\text { histogram (LBPH) Classifier }\end{array}$ & $\begin{array}{l}\text { Achieved accuracy about } \\
93.67 \%\end{array}$ \\
\hline
\end{tabular}

\subsection{Smart Agriculture}

There has been ever-increasing demand for food supplies due to exponential growth in the world population. Conventional methods alone might not be able to keep up with this demand. Smart agriculture which is considered as one of the few realistic ways, smart agriculture integrates the use of different technologies to better monitor growth crop yield prediction, plant disease detection, weed detection, irrigation management, prediction of soil properties etc. Smart agriculture happens to be one of the many disciplines where use of deep learning and computer vision are being realized to be of major impact. The use of technology in relation to smart agriculture should enable transmission of correct and accurate information to farmers at right time.

Figure 3 shows popular application of Computer Vision and Deep Learning in Agriculture like plant disease detection wherein accurate diagnosis of probable plant disease can save the entire crop from getting infected, fruit counting and yield production can help farmers make necessary packing and storage requirements before sale, weather prediction to minimize crop loss due to severe weather conditions and crop type classification to identify variety of crops using deep learning models.

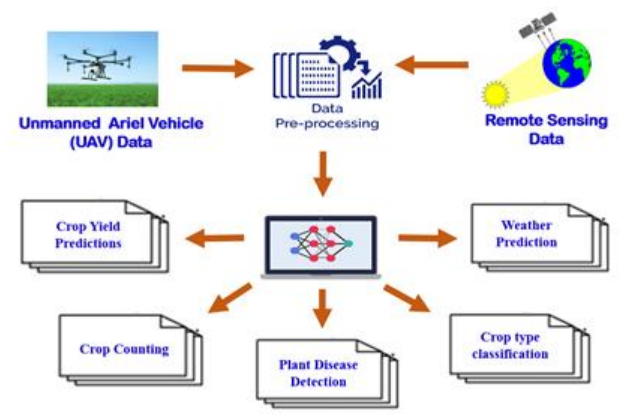

Figure 3 Popular Applications of Computer Vision and Deep Learning in Agriculture

Table 2. Major application areas of Computer Vision and Deep Learning in Agriculture

\begin{tabular}{|c|l|l|l|l|}
\hline \multirow{2}{*}{$\begin{array}{c}\text { Category } \\
\text { Plant disease } \\
\text { detection }\end{array}$} & $\begin{array}{l}\text { Reference } \\
\text { (2020) }\end{array}$ & \multicolumn{1}{|c|}{ Application } & \multicolumn{1}{c|}{ DL Model Used } & Dataset \\
\cline { 2 - 5 } & & $\begin{array}{l}\text { Detection of healthy } \\
\text { leaves and thirteen } \\
\text { different diseases }\end{array}$ & $\begin{array}{l}\text { Alexnet, VGG, } \\
\text { Inception (Modified) }\end{array}$ & $\begin{array}{l}\text { Plant village } \\
\text { dataset }\end{array}$ \\
\hline
\end{tabular}




\begin{tabular}{|c|c|c|c|c|}
\hline & $\begin{array}{l}\text { Konstantinos et al. } \\
\text { [53] (2018) }\end{array}$ & $\begin{array}{l}\text { Detection of plant } \\
\text { disease for } 25 \text { plants. }\end{array}$ & $\begin{array}{l}\text { AlexNet, } \\
\text { AlexNetOWTBn, } \\
\text { GoogleNet, } \\
\text { Overfeat,VGG }\end{array}$ & $\begin{array}{l}\text { Plant village } \\
\text { dataset }\end{array}$ \\
\hline & $\begin{array}{l}\text { Mohanty et al.[54] } \\
\text { (2016) }\end{array}$ & $\begin{array}{l}\text { Identification of } 14 \text { crop } \\
\text { species and } 26 \text { diseases }\end{array}$ & Deep CNN & $\begin{array}{l}\text { Plant village } \\
\text { dataset }\end{array}$ \\
\hline \multirow[t]{3}{*}{ Crop Count } & $\begin{array}{l}\text { Joao Valente et al. } \\
\text { [55] (2020) }\end{array}$ & $\begin{array}{l}\text { To detect number of } \\
\text { plants on the field that } \\
\text { emerged after sowing. }\end{array}$ & Alexnet & $\begin{array}{l}\text { UAV-acquired } \\
\text { spinach images } \\
\text { from field of } \\
\text { Netherland }\end{array}$ \\
\hline & $\begin{array}{l}\text { Bruno et al. [56] } \\
\text { (2019) }\end{array}$ & $\begin{array}{l}\text { To detect number of } \\
\text { plants on the field that } \\
\text { emerged after sowing. } \\
\text { Corn Plant Counting }\end{array}$ & U-Net & $\begin{array}{l}\text { UAV acquired } \\
\text { images }\end{array}$ \\
\hline & Steven et al.[57] (2017) & Orange and Apple count & $\begin{array}{l}\text { Neural Network + } \\
\text { Linear Regression }\end{array}$ & $\begin{array}{l}\text { UAV acquired } \\
\text { images }\end{array}$ \\
\hline \multirow[t]{3}{*}{ Weather Prediction } & $\begin{array}{l}\text { Ashesh et al.[58] } \\
\text { (2020) }\end{array}$ & $\begin{array}{l}\text { prediction of heat waves } \\
\text { and cold spells }\end{array}$ & $\begin{array}{l}\text { ConvNet and } \\
\text { CapsNet }\end{array}$ & LENS dataset \\
\hline & $\begin{array}{l}\text { Snehlata et al.[59] } \\
\text { (2020) }\end{array}$ & Cyclone Prediction & $\begin{array}{l}\text { Xception, } \\
\text { NasNetMobile, and } \\
\text { Mobile Net }\end{array}$ & $\begin{array}{l}\text { IMD, MOSDAC } \\
\text { and KALPANA-I } \\
\text { satellite images }\end{array}$ \\
\hline & $\begin{array}{l}\text { Renato et al.[60] } \\
\text { (2019) }\end{array}$ & $\begin{array}{l}\text { Crop Yield prediction } \\
\text { based on crop genotype, } \\
\text { environment and their } \\
\text { interactions. }\end{array}$ & Neural network & $\begin{array}{l}2018 \text { Syngenta } \\
\text { Crop Challenge }\end{array}$ \\
\hline \multirow[t]{2}{*}{ Crop Yield Prediction } & Peteri et al.[61] (2019) & $\begin{array}{l}\text { Crop yield prediction of } \\
5 \text { crops: Corn, Cotton, } \\
\text { Rice, Soyabean, } \\
\text { Sugarcane }\end{array}$ & DNN + LSTM & $\begin{array}{l}\text { Produo Agrcola } \\
\text { Municipal (PAM) }\end{array}$ \\
\hline & Anna et al.[62] (2019) & $\begin{array}{l}\text { Prediction of wheat and } \\
\text { barley yield }\end{array}$ & $\begin{array}{l}\text { Customised CNN } \\
\text { model with } \\
\text { Adadelta training } \\
\text { algorithm }\end{array}$ & $\begin{array}{l}\text { UAV-acquired } \\
\text { multispectral } \\
\text { data from } 9 \\
\text { fields. }\end{array}$ \\
\hline
\end{tabular}




\begin{tabular}{|l|l|l|l|l|}
\hline $\begin{array}{c}\text { Crop Type } \\
\text { Classification }\end{array}$ & $\begin{array}{l}\text { Yang-yang et al. [63] } \\
(2020)\end{array}$ & $\begin{array}{l}\text { Classifying 31 classes of } \\
\text { fruits and vegetables } \\
\text { using various DL models }\end{array}$ & $\begin{array}{l}\text { VGG16, VGG19, } \\
\text { SqueezeNet, } \\
\text { InceptionV4, } \\
\text { DenseNet121, } \\
\text { ResNet18, ResNet50 }\end{array}$ & $\begin{array}{l}\text { CropDeep } \\
\text { dataset }\end{array}$ \\
\cline { 2 - 5 } & $\begin{array}{l}\text { Liheng et al.[64] } \\
\text { (2019) }\end{array}$ & $\begin{array}{l}\text { Classification of } 14 \\
\text { summer crops }\end{array}$ & $\begin{array}{l}\text { Conv1D + LSTM } \\
\text { dataset }\end{array}$ \\
\hline
\end{tabular}

\section{CHALLENGES}

Challenges in smart cities are elaborated here: (i) accuracy and robustness in computer vision and sensor network, enhancement in result with high accuracy over object detection, object recognition, object classification, segmentation, transform learning etc. are required. Robustness is a major concern in smart cities because of different geographical conditions. Atmosphere and weather conditions are different for various geographical area, like areas covered with mountains, harbour, dusty etc. so more accuracy and enhancement in technique is required to utilize services in real time. (ii) Cybersecurity and privacy are also challenging tasks in smart cities. Internet connected devices generate and transmit huge chunks of data, privacy for data which may be relevant to CCTV, medical diagnosis, fund transfer, gas station or charging station, electricity or power supply, food supply, emergency services and many more. If there is a case, criminals easily gain access to data and use for illegal activity. Hence the government and IT support system should strengthen enough to prevent cyberattack. (iii) Infrastructure, well equipped resources are required to install and utilize smart technology like Artificial Intelligent, Computer vision, IoT. (iv) Engaging the community, smart city truly exists, when citizens are engaged and actively involved in new projects and practices.

\section{CONCLUSION}

Computer Vision is used to provide better quantitative information that is unobtainable subjectively, leading to the eventual replacement of human effort. Computer Vision is a necessary and promising one for analysing the qualities and information using different convolution networks like AlexNet, ZFNet, VGG-19, ResNet etc. These model learning systems are an essential part of feature extraction. Deep learning frameworks are a cornerstone for Computer Vision and used in variety of visual understanding tasks, such as object detection, face recognition, action and activity recognition, human pose estimation, image retrieval, and semantic segmentation. The most applicability of Computer Vision is in smart cities involving new use cases related to smart healthcare, smart transportation, video surveillance and smart agriculture. These are among the most important open issues in Smart City that attract the interest of the research community in Computer Vision.

\section{REFERENCES}

[1] Vinayakumar R SSQVPSKP Mamoun Alazab. A Visualized Botnet Detection System based Deep Learning for the Internet of Things Networks of Smart Cities. IEEE Transactions on Industry Applications 2020.

[2] N Doulamis AD, Protopapadakis E. Deep Learning for Computer Vision: A Brief Review 2018. [3] C. Baier, J-P. Katoen, Principles of Model Checking, MIT Press, 2008.

[3] Bhuvaneswary, N., S. Prabu, S. Karthikeyan, R. Kathirvel, and T. Saraswathi. "Low Power Reversible Parallel and Serial Binary Adder/Subtractor." Further Advances in Internet of Things in Biomedical and Cyber Physical Systems (2021): 151.

[4] Prabu, S., Balamurugan Velan, F. V. Jayasudha, P. Visu, and K. Janarthanan. "Mobile technologies for contact tracing and prevention of COVID-19 positive cases: a cross-sectional study." International Journal of Pervasive Computing and Communications (2020).

[5] Y. Sun, J. Liu, K. Yu, M. Alazab, K. Lin, "PMRSS: Privacy-preserving Medical Record Searching Scheme for Intelligent Diagnosis in IoT Healthcare", IEEE Transactions on Industrial Informatics, doi: 10.1109/TII.2021.3070544.

[6] Z. Guo, L. Tang, T. Guo, K. Yu, M. Alazab, A. Shalaginov, "Deep Graph Neural Network-based Spammer Detection Under the Perspective of Heterogeneous Cyberspace", Future Generation Computer Systems.

[7] Rajendrakumar, Shiny, and V. K. Parvati. "Automation of irrigation system through 
embedded computing technology." In Proceedings of the 3rd International Conference on Cryptography, Security and Privacy, pp. 289-293. 2019.

[8] Kumar, M. Keerthi, B. D. Parameshachari, S. Prabu, and Silvia liberata Ullo. "Comparative Analysis to Identify Efficient Technique for Interfacing BCI System." In IOP Conference Series: Materials Science and Engineering, vol. 925, no. 1, p. 012062. IOP Publishing, 2020.

[9] Subramani, Prabu, K. Srinivas, R. Sujatha, and B. D. Parameshachari. "Prediction of muscular paralysis disease based on hybrid feature extraction with machine learning technique for COVID-19 and post-COVID-19 patients." Personal and Ubiquitous Computing (2021): 1-14.

[10] Naeem, Muhammad Ali, Tu N. Nguyen, Rashid Ali, Korhan Cengiz, Yahui Meng, and Tahir Khurshaid. "Hybrid Cache Management in IoTbased Named Data Networking." IEEE Internet of Things Journal (2021).

[11] Le, Ngoc Tuyen, Jing-Wein Wang, Duc Huy Le, Chih-Chiang Wang, and Tu N. Nguyen. "Fingerprint enhancement based on tensor of wavelet subbands for classification." IEEE Access 8 (2020): 6602-6615.

[12] Do, Dinh-Thuan, Tu Anh Le, Tu N. Nguyen, Xingwang $\mathrm{Li}$, and Khaled M. Rabie. "Joint impacts of imperfect CSI and imperfect SIC in cognitive radio-assisted NOMA-V2X communications." IEEE Access 8 (2020): 128629-128645.

[13] Alazab T M Tang. Deep Learning Applications for Cyber Security segmentation and feature selection. Advanced Sciences and Technologies for Security Applications, Springer International 2019.

[14] Farhan Ullah SJFATMA Junfeng Wang. Source Code Authorship Attribution Using Hybrid Approach of Program Dependence Graph and Deep Learning Model 2019.

[15] C Szegedy SIJSZW V Vanhoucke. Rethinking the inception architecture for computer vision 2016.

[16] Anjali Goel "Lane Detection Techniques - A Review", International Journal of Computer Science and Mobile Computing, International Journal of Computer Science and Mobile Computing - 2014.

[17] Yeongmin Ko et al."Key Points Estimation and Point Instance Segmentation Approach for Lane Detection" Submitted to "IEEE Transactions on Intelligent Transportation Systems 2020.
[18] Y. Hou, Z. Ma2, C. Liu2, C Change Loy "Learning Lightweight Lane Detection CNNs by Self Attention Distillation”. ICCV 2019.

[19] A. Revelo, R. Álvarez and F. Grijalva, "Human Drowsiness Detection In Real Time, Using Computer Vision," 2019 IEEE Fourth Ecuador Technical Chapters Meeting (ETCM), Guayaquil, Ecuador, pp. 1-6, doi: 10.1109/ETCM48019.2019.9014884, 2019.

[20] Nataniel Ruiz, Eunji Chong James, M. Rehg "Fine-Grained Head Pose EstimationWithout Keypoints", Accepted to Computer Vision and Pattern Recognition Workshops (CVPRW), IEEE Conference, 2018.

[21] Wang, Haofan \& Chen, Zhenghua \& Zhou, Yi., Hybrid coarse-fine classification for head pose estimation, 2019.

[22] Yang, Tsun-Yi, et al. "Fsa-net: Learning finegrained structure aggregation for head pose estimation from a single image." Proceedings of the IEEE Conference on Computer Vision and Pattern Recognition, 2019.

[23] C. Tang et al. "Improving Pedestrian Attribute RecognitionWith Weakly-Supervised Multi-Scale Attribute-Specific Localization" Accepted by ICCV 2019.

[24] Liu, Xihui \& Zhao, Haiyu \& Tian, Maoqing \& Sheng, Lu \& Shao, Jing \& Yi, Shuai \& Yan, Junjie \& Wang, Xiaogang. (2017). HydraPlus-Net: Attentive Deep Features for Pedestrian Analysis. 350-359. 10.1109/ICCV.2017.46, 2017.

[25] Tabernik, Domen, and Danijel Skočaj. "Deep learning for large-scale traffic-sign detection and recognition." IEEE Transactions on Intelligent Transportation Systems 21.4 (2019): 1427-1440, 2019.

[26] Dollár, Piotr \& Wojek, Christian \& Schiele, Bernt \& Perona, Pietro. (2011). Pedestrian Detection: An Evaluation of the State of the Art. IEEE transactions on pattern analysis and machine intelligence. $34 . \quad 743-61$. 10.1109/TPAMI.2011.155, 2011.

[27] Felzenszwalb, Pedro \& Girshick, Ross \& Mcallester, David \& Ramanan, Deva. Object Detection with Discriminatively Trained PartBased Models. IEEE transactions on pattern analysis and machine intelligence. 32. 1627-45. 10.1109/TPAMI.2009.167, 2010.

[28] A. Arinaldi, et al. "Detection and classification of vehicles for traffic video analytics" INNS Conference on Big Data and Deep Learning, 2018.

[29] Lee, Seokju, et al. "Vpgnet: Vanishing point guided network for lane and road marking detection and recognition." Proceedings of the 
IEEE international conference on computer vision, 2017.

[30] Fan Zhang, et al. DetReco: Object-Text Detection and Recognition Based on Deep Neural Network, Mathematical Problems in Engineering Volume 2020, Article ID 2365076 -2020.

[31] O. Shobayo, et al. Development of Smart Plate Number Recognition System for Fast Cars with Web Application, Applied Computational Intelligence and Soft Computing Volume 2020, Article ID 8535861, 2020

[32] Barringer, R. Kuiper, A. Pnueli, Now you may compose temporal logic specifications, in: Proceedings of the Sixteenth Annual ACM Symposium on the Theory of Computing (STOC), ACM, 1984, pp. 51-63. DOI:

[33] Xinyue Yang, Xiaoyang Ren, Meng Chen, Luqi Wang, Yuhao Ding. "Human Posture Recognition in Intelligent Healthcare", Journal of Physics: Conference Series, 2020.

[34] Yilmaz A, Javed O, Shah M (2006) Object tracking: a survey. ACM Comput Surv 38(4):13, 2006.

[35] L. Tan, K. Yu, F. Ming, X. Cheng, G. Srivastava, "Secure and Resilient Artificial Intelligence of Things: a HoneyNet Approach for Threat Detection and Situational Awareness", IEEE Consumer Electronics Magazine, 2021, doi: 10.1109/MCE.2021.3081874.

[36] L. Tan, N. Shi, K. Yu, M. Aloqaily, Y. Jararweh, "A Blockchain-Empowered Access Control Framework for Smart Devices in Green Internet of Things", ACM Transactions on Internet Technology, vol. 21, no. 3, pp. 1-20, 2021,https://doi.org/10.1145/3433542.

[37] Z. Guo, A. K. Bashir, K. Yu, J. C. Lin, Y. Shen, "Graph Embedding-based Intelligent Industrial Decision for Complex Sewage Treatment Processes", International Journal of Intelligent Systems, 2021, doi: 10.1002/int.22540.

[38] Z. Guo, K. Yu, A. Jolfaei, A. K. Bashir, A. O. Almagrabi, and N. Kumar, "A Fuzzy Detection System for Rumors through Explainable Adaptive Learning”, IEEE Transactions on Fuzzy Systems, doi: 10.1109/TFUZZ.2021.3052109.

[39] Tian Y, Senior A, Lu M (2012) Robust and efficient foreground analysis in complex surveillance videos. Mach Vis Appl 23(5):967983, 2012 .

[40] Zin TT, Tin P, Toriu T, Hama H (2012a) A novel probabilistic video analysis for stationary object detection in video surveillance systems. IAENG Int J Comput Sci 39(3):295-306, 2012
[41] Fan Q, Pankanti S (2012) Robust foreground and abandonment analysis for large-scale abandoned object detection in complex surveillance videos. In: IEEE ninth international conference on advanced video and signal- based surveillance (AVSS), IEEE, pp 58-63, 2012.

[42] SanMiguel J, Caro L, Martinez J, Pixel-based colour contrast for abandoned and stolen object discrimination in video surveillance. Electron Lett 48(2):86-87, 2012.

[43] Tripathi RK, Jalal AS, Bhatnagar C (2013) A framework for abandoned object detection from video surveil- lance. In: Fourth national conference on computer vision, pattern recognition, image processing and graphics (NCVPRIPG), IEEE, pp 1-4, 2013

[44] Sajith K, Nair KR (2013) Abandoned or removed objects detection from surveillance video using codebook. Int J Eng Res Technol 2:401-406, 2013.

[45] Ferryman J, Hogg D, Sochman J, Behera A, Rodriguez-Serrano JA, Worgan S, Li L, Leung V, Evans M, Cornic P et al (2013) Robust abandoned object detection integrating wide area visual surveillance and social context. Pattern Recogn Lett 34(7):789-798, 2013.

[46] Chitra M, Geetha MK, Menaka L, et al (2013) Occlusion and abandoned object detection for surveillance applications. Int J Comput Appl Technol Res 2(6):708- meta, 2013.

[47] Nam Y (2016) Real-time abandoned and stolen object detection based on spatiotemporal features in crowded scenes. Multimed Tools Appl 75(12):7003-7028, 2016.

[48] Virender Singha, Swati Singha, Dr. Pooja Gupta, Real-Time Anomaly Recognition Through CCTV Using Neural Networks, International Conference on Smart Sustainable Intelligent Computing and Applications under ICITETM2020, 2020.

[49] Amrutha C.V, C. Jyotsna, Amudha J., Deep Learning Approach for Suspicious Activity Detection from Surveillance Video, Proceedings of the Second International Conference on Innovative Mechanisms for Industry Applications (ICIMIA 2020) IEEE Xplore Part Number: CFP20K58-ART; ISBN: 978-1-7281-4167-1, 2020.

[50] Rohit Rastogi, Rishabh Jain, Puru Jain, Parul Singhal, Priyanshi Garg and Mukund Rastogi, Inference-Based Statistical Analysis for Suspicious Activity Detection Using Facial Analysis, Computational Intelligence in Pattern Recognition, Proceedings of CIPR, 2020

[51] Arvind Jaiswal, Sandhya Tarar, Real-Time Biometric system for security and surveillance 
using face recognition, 4th International Conference, ICACDS 2020 ,Valletta, Malta, April 24-25, 2020.

[52] Shradha S. Pradhan and Rupali Patil, Comparison of Deep Learning Approaches for Plant Disease Detection, H. Vasudevan et al. (eds.), Proceedings of International Conference on Wireless Communication, Lecture Notes on Data Engineering and Communications Technologies, Springer Nature Singapore Pte Ltd, 2020.

[53] Konstantinos P.Ferentinos, Deep learning models for plant disease detection and diagnosis, Computers and Electronics in Agriculture Volume 145, February 2018, Pages 311-318

[54] Mohanty, S.P., Hughes, D.P., Salathé, M., 2016. Using deep learning for image-based plant disease detection. Front. Plant. Sci. 7.

[55] Joao Valente, Bilal Sari, Lammert Kooistra, Henk Kramer, Sander Mucher, Automated crop plant counting from very high-resolution aerial imagery, Precision Agriculture, 2020.

[56] Bruno T. Kitano, Caio C. T. Mendes, André R. Geus, Henrique C. Oliveira, and Jefferson R. Souza, Corn Plant Counting Using Deep Learning and UAV Images, IEEE GEOSCIENCE AND REMOTE SENSING LETTERS, 2019.

[57] Steven W Chen, Shreyas S. Shivakumar, Sandeep Dcunha, Jnaneshwar Das, Edidiong Okon, Chao $\mathrm{Qu}$, Camillo J. Taylor, and Vijay Kumar, Counting Apples and Oranges with Deep Learning: A Data Driven Approach, IEEE ROBOTICS AND AUTOMATION LETTERS, 2017.

[58] Ashesh Chattopadhyay Ebrahim Nabizadeh Pedram Hassanzadeh, Analog Forecasting of Extreme-Causing Weather Patterns Using Deep
Learning, Journal of Advances in Modeling Earth Systems, Feb, 2020

[59] Snehlata Shakya , Sanjeev Kumar, and Mayank Goswami, Deep Learning Algorithm for Satellite Imaging Based Cyclone Detection, IEEE JOURNAL OF SELECTED TOPICS IN APPLIED EARTH OBSERVATIONS AND REMOTE SENSING, VOL. 13, 2020

[60] Renato Luiz de Freitas Cunha, Bruno Silva, ESTIMATING CROP YIELDS WITH REMOTE SENSING AND DEEP LEARNING, Latin American GRSS \& ISPRS Remote Sensing Conference, 2020 ( arXiv:2007.10882v1).

[61] Petteri Nevavuori, Nathaniel Narraa Tarmo Lippinga, Crop yield prediction with deep convolutional neural networks, Computers and Electronics in Agriculture, Volume 163, 104859, August 2019.

[62] Anna X. Wang, Caelin Tran, Nikhil Desai, David Lobell, Stefano Ermon, Deep Transfer Learning for Crop Yield Prediction with Remote Sensing Data, Proceedings of the 1st ACM SIGCAS Conference on Computing and Sustainable Societies, June 2018.

[63] Yang-Yang Zheng, Jian-Lei Kong ,Xue-Bo Jin, Xiao-Yi Wang,Ting-Li Su and Min Zuo, CropDeep: The Crop Vision Dataset for DeepLearning-Based Classification and Detection in Precision Agriculture, Internet-of-Things for Precision Agriculture (IoAT)), Feb 2020.

[64] Liheng Zhonga, Lina Hub , Hang Zhouc, Deep learning based multi-temporal crop classification, Remote Sensing of Environment, 2019. 